\title{
Effect of Diode Low-level Laser Irradiation Time on Socket Healing
}

\author{
Ali Peimani ${ }^{1}$, Tahereh Eslammanesh ${ }^{2}$, Mostafa Sadeghi ${ }^{3}$, Ramin Rahati $^{4}$
}

\begin{abstract}
Aim: The purpose of this research was to survey the effect of low-level laser irradiation time on socket healing in rats. Materials and methods: This randomized nonblinded animal study was done on 24 male rats that were divided into four groups. First maxillary molars of rats were extracted through general anesthesia, and laser was used in all four groups: first group with zero radiation time, second group with 3 minutes, third group with 5 minutes, and fourth group with 10 minutes of radiation by the diode laser (power: $100 \mathrm{~mW}$, wavelength: $980 \mathrm{~nm}$ ). Half of the rats (three rats) were sacrificed on the 3rd day and another half of rats were sacrificed on the 7th day. Then, the presence of angiogenesis, bone trabeculae, fibroblasts, neutrophil cells, macrophage cells, and lymphocyte cells was assessed. Data were analyzed by SPSS (version 21) using parametric tests.

Results: Among 24 rats, on the 3rd day, the percentage of macrophage and bone trabecula increased significantly in the 5 minute group ( $p=$ 0.041 and $p<0.01$, respectively). Other changes in days 3 and 7 were not statistically significant $(p>0.05)$.

Conclusion: Low-level laser radiation can accelerate the process of tooth socket healing, which was particularly noticeable in the 5 minute radiation over 3 days.

Clinical significance: Using a low-level laser can be helpful in accelerating the healing of the tooth socket and reduce the complications after tooth extraction.

Keywords: Laser irradiation, Low-level laser therapy, Tooth socket, Wound healing.

The Journal of Contemporary Dental Practice (2020): 10.5005/.jp-journals-10024-2862
\end{abstract}

\section{INTRODUCTION}

Tooth extraction is the most common surgical procedure performed by dentists that leaves an open wound in the soft and bone tissues. Different materials and methods are used to accelerate wound healing in the soft and bone tissues. ${ }^{1,2}$

Wounds involving oral mucosa and alveolar bones are commonly found in oral surgical treatments. ${ }^{3}$ The healing of these wounds depends on the severity of the surgery; however, healing some wounds requires a long time and can cause more discomfort to patients. ${ }^{4}$ Methods such as topical or systemic corticosteroids, nonsteroidal anti-inflammatory drugs, drains, various surgical cutouts, and low-level laser can be used to prevent the complications of tooth extraction. ${ }^{5}$

Low-level laser is one of the new methods of bone healing that has some benefits such as reduction of pain and inflammation, stimulation of collagen production, and cell proliferation. ${ }^{6}$ Lowlevel lasers also affect the activity of bone cells. An increase has also been reported in the superficial osteoblasts and the thickness of osteoid cells in the laser radiation region. ${ }^{7}$ Park et al. ${ }^{8}$ reported that laser therapy has a time-dependent effect. Pretel et al. ${ }^{9}$ also showed the effect of laser radiation on the bone defects healed on days 15 and 45 . But Lopez-Ramirez et al. ${ }^{10}$ stated that low-level laser did not affect the direction of pain, swelling, and trismus after mandibular third molar surgery.

Although the use of low-level laser is one of the known methods for accelerating tooth socket healing and preventing its complications, few studies have been done on the physical parameters of the laser, including the time of radiation. Therefore, the purpose of this research was to investigate the effect of low-level laser irradiation time on socket healing in rats. If tooth socket healing accelerates, a suitable time for laser radiation will be advised.
${ }^{1}$ Department of Oral Surgery, Dental School of Rafsanjan University, Rafsanjan, Iran

${ }^{2}$ Department of Pathology, Clinical Researches Development Unit, AliIbn Abitaleb Hospital, Medical School, Rafsanjan University of Medical Sciences, Rafsanjan, Iran

${ }^{3}$ Department of Operative Dentistry, Dental School of Rafsanjan University, Rafsanjan, Iran

${ }^{4}$ Rafsanjan University of Medical Sciences, Rafsanajan, Iran

Corresponding Author: Ramin Rahati, Rafsanjan University of Medical Sciences, Rafsanajan, Iran, Phone: +98 9157648401, e-mail: Ramin. rahati@yahoo.com

How to cite this article: Peimani A, Eslammanesh T, Sadeghi M, et al. Effect of Diode Low-level Laser Irradiation Time on Socket Healing. J Contemp Dent Pract 2020;21(6):640-644.

Source of support: This research was supported by the Rafsanajn University of Medical Sciences

Conflict of interest: None

\section{Materials and Methods}

\section{Animals}

In this experimental study, 22 male Wistar rats $(250-300 \mathrm{~g})$ were kept in separate standard cages with free access to water and food. Ethical confirmation of animal methods from the Institutional Animal Care was obtained by the Committee of Rafsanjan University of Medical Sciences, and all experiments were carried out with the least suffering of animals and with the smallest possible number of animals for validated statistical evaluation. Furthermore, the animals were examined by a vet 


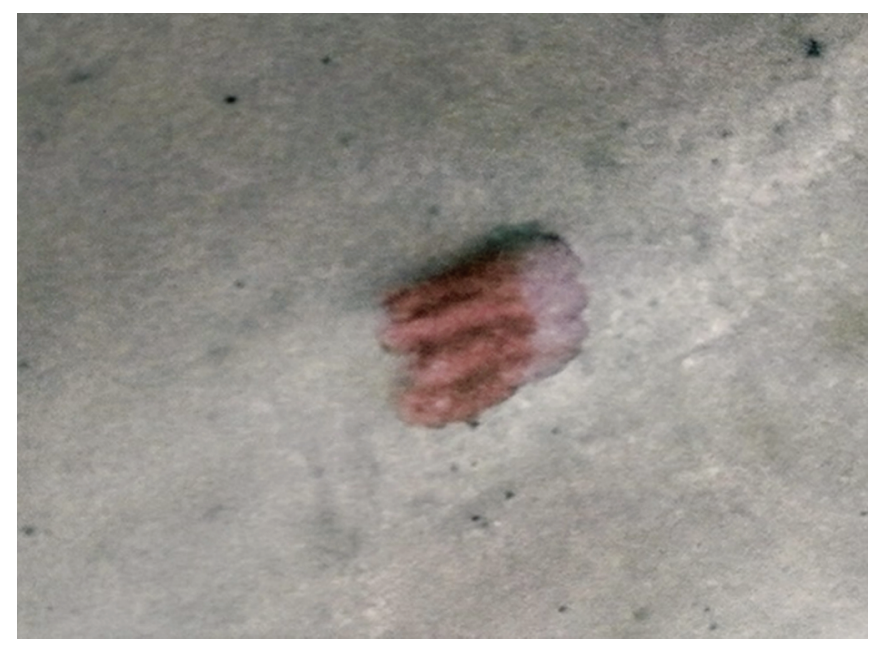

Fig. 1: Extracted molar. Maxillary first molar was extracted by surgical forceps with minimum damage

before the work and were examined for doing surgery and general health.

\section{Surgical Procedures}

The samples were subjected to anesthesia with an intraperitoneal injection of 0/6 cc ketamine 5\% (Alfasan, Amsterdam, Netherland) with the rate of $100 \mathrm{mg} / \mathrm{kg}$ and xylacin 2\% (Alfasan, Amsterdam, Netherland) with 1:9 ratio. The maxillary first molar was extracted by surgical forceps with minimum damage (Fig. 1). ${ }^{11,12}$

\section{Laser Irradiation}

According to Park et al., ${ }^{8}$ the animals were accidentally divided into four groups of six according to the time of radiation. The first group was not exposed to laser radiation, the second group received 3 minutes radiation a day, the third group received 7 minutes radiation a day, and the fourth group received 10 minutes radiation a day. Immediately after controlling the hemorrhage, a laser diode (Doctor smile, Genoa, Italy) was used with the maximum power of $100 \mathrm{~mW}$ and a wavelength of $980 \mathrm{~nm}$ for 3, 5, and 10 minutes in the mouth, with a minimum laser probe distance from the tooth socket and no contact 3 or 7 days after tooth extraction. In the control group, too, the laser probe was set like some other groups but the laser was not activated (Fig. 2).

\section{Sample Preparation}

The rats were kept in single cages after recovery by considering the surgery day as day 0 . Three rats from each group after 3 days and three other rats after 7 days were randomly sacrificed.

\section{Histological Procedures}

The separated heads were fixed in 10\% formalin (Kolanky, Henan, China) at $\mathrm{pH} 7$ and were then placed in the paraffin blocks. After 48 hours, the maxilla of the rats was removed, fixed, and demineralized in 4\% EDTA (Merck, Berlin, Germany). After 3 weeks, complete bone tissue softening was tested by a surgical knife. ${ }^{13}$

The specimens were dehydrated in alcohol and xylene, put in paraffin, and cut serially into $5 \mu \mathrm{m}$ sections by a microtome (SIEE Medial, Mainz, Germany).

Next, they were stained with $\mathrm{H}$ and $\mathrm{E}$. A blinded to the experimental design observer, in a division with two pathologists,

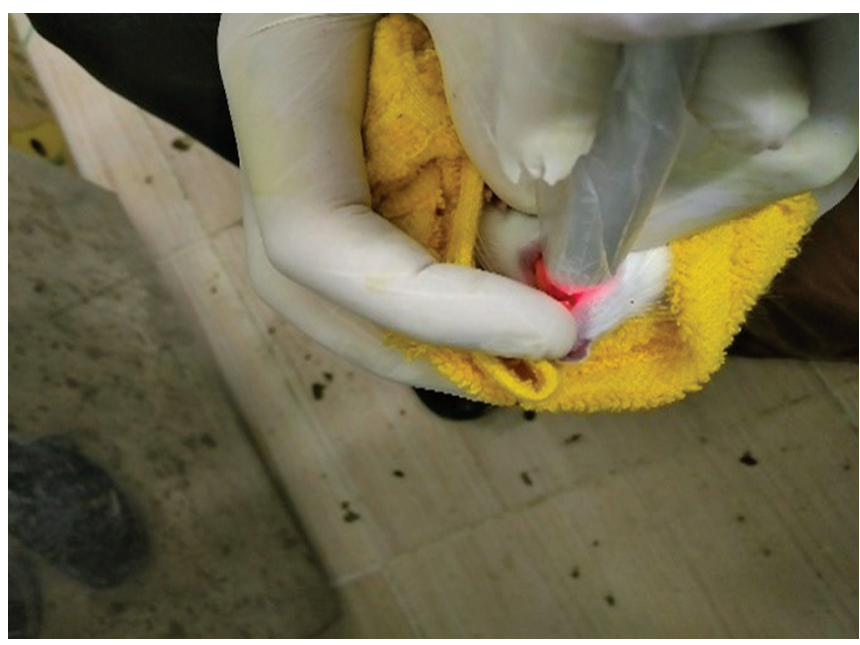

Fig. 2: Laser application. Laser applicated with a minimum laser probe distance from the tooth socket

evaluated the histopathological variables of the maxilla after standardization of the measurements.

The presence of angiogenesis, bone trabeculae, fibroblasts, neutrophil cells, macrophage cells, and lymphocyte cells were assessed by the CXR2 optic microscope (Labomed, Los Angeles, USA). For the stained samples, counting tables were drawn and all samples were observed under the microscope and the number of each variable was recorded in these tables.

\section{Statistical Analysis}

All statistical analyzes were performed by the SPSS software (version 21; SPSS Inc., Chicago, IL). The data were presented, and statistically significant differences among the groups were compared using the Shapiro-Wilk, two-way repeated measure ANOVA, and Bonferroni tests. $p$ values less than 0.05 were considered to indicate statistical significance.

\section{Results}

The data distribution was normal according to the ShapiroWilk test ( $p$ value $>0.05$ ). The mean and standard deviation of histopathologic variables were calculated in all study groups on the 3rd and 7th days by this test (Table 1).

As shown in Table 2, the parametric Bonferroni test was used to compare the case groups with the control group. The results of this test showed that the mean of macrophages and bone trabeculae increased significantly in the 5 minute daily radiation group on the 3 rd day compared to the control group ( $p$ value $<0.05$ ). The fibroblasts in all groups of laser radiation increased in comparison with the control group, which was the highest in 5 minute daily radiation group, but the differences were not statistically significant ( $p$ value $>0.05$ ). The neutrophils also decreased in all groups of laser radiation compared to the control group. The highest decrease was seen in 5 minute daily radiation group, but these differences were not statistically significant ( $p$ value $>0.05$ ). Other variables were not statistically significant in comparison with the control group ( $p$ value $>0.05$ ) (Fig. 3).

On the 7th day, the amount of bone trabeculae increased in all laser radiation groups and the number of lymphocytes decreased compared to the control group, but it was not statistically significant according to the Bonferroni statistical test ( $p$ value $>$ 
Effect of Diode Laser on Socket Healing

Table 1: Mean and standard deviation of histopathologic variables in four studied groups

\begin{tabular}{|c|c|c|c|c|c|}
\hline \multirow{2}{*}{$\begin{array}{l}\text { Histopathological vari- } \\
\text { ables }\end{array}$} & \multirow[b]{2}{*}{ Day } & Control & 3 minutes radiation & 5 minutes radiation & 10 minutes radiation \\
\hline & & \multicolumn{4}{|c|}{ Mean \pm standard deviation } \\
\hline \multirow[t]{2}{*}{ Bone trabeculae } & Third & $4.1 \pm 17.35$ & $13.4 \pm 52.62$ & $44.8 \pm 92.63$ & $18.11 \pm 83.19$ \\
\hline & Seventh & $30.1 \pm 31.14$ & $31.9 \pm 20.40$ & $48.8 \pm 95.06$ & $49.1 \pm 75.72$ \\
\hline \multirow[t]{2}{*}{ Fibroblasts } & Third & $8.2 \pm 0.64$ & $12.2 \pm 33.08$ & $19.9 \pm 33.45$ & $8.5 \pm 33.78$ \\
\hline & Seventh & $23.9 \pm 0.16$ & $23.4 \pm 33.93$ & $37.4 \pm 33.16$ & $21.8 \pm 33.14$ \\
\hline \multirow[t]{2}{*}{ Neutrophil cells } & Third & $68.4 \pm 0.00$ & $51.13 \pm 67.50$ & $41.16 \pm 67.63$ & $61.10 \pm 67.40$ \\
\hline & Seventh & $27.7 \pm 66.37$ & $38.5 \pm 67.50$ & $26.5 \pm 33.13$ & $33.13 \pm 33.65$ \\
\hline \multirow[t]{2}{*}{ Macrophage cells } & Third & $13.2 \pm 67.08$ & $22.8 \pm 0.54$ & $28.2 \pm 0.00$ & $19.3 \pm 0.60$ \\
\hline & Seventh & $37.6 \pm 33.42$ & $27.5 \pm 00.20$ & $27.3 \pm 33.79$ & $27.2 \pm 66.51$ \\
\hline \multirow[t]{2}{*}{ Lymphocyte cells } & Third & $10.0 \pm 33.58$ & $14.4 \pm 00.58$ & $11.6 \pm 0.55$ & $11.1 \pm 0.73$ \\
\hline & Seventh & $12.6 \pm 0.55$ & $12.1 \pm 0.00$ & $9.2 \pm 0.00$ & $6.3 \pm 67.51$ \\
\hline \multirow[t]{2}{*}{ Angiogenesis } & Third & $12.2 \pm 33.52$ & $12.2 \pm 67.52$ & $10.5 \pm 33.03$ & $8.3 \pm 67.51$ \\
\hline & Seventh & $13.4 \pm 33.41$ & $13.2 \pm 67.09$ & $11.2 \pm 66.08$ & $9.1 \pm 33.15$ \\
\hline
\end{tabular}

Table 2: Comparison of histopathologic variables of the studied groups with the control group on the 3rd and 7th days

\begin{tabular}{|c|c|c|c|c|c|c|}
\hline \multirow{2}{*}{$\begin{array}{l}\text { Histopathological } \\
\text { variables }\end{array}$} & \multicolumn{3}{|c|}{$3 r d$ day } & \multicolumn{3}{|c|}{ 7th day } \\
\hline & 3 minutes radiation & 5 minutes radiation & 10 minutes radiation & & 5 minutes radiation & 10 minutes radiation \\
\hline Bone trabeculae & 0.981 & $0.001^{*}$ & 0.257 & 0.998 & 0.127 & 0.998 \\
\hline Fibroblasts & 0.998 & 0.260 & 0.998 & 0.998 & 0.210 & 0.998 \\
\hline Neutrophil cells & 0.817 & 0.170 & 0.998 & 0.0941 & 0.998 & 0.273 \\
\hline Macrophage cells & 0.413 & 0.041 & 0.998 & 0.167 & 0.191 & 0.281 \\
\hline Lymphocyte cells & 0.998 & 0.998 & 0.998 & 0.998 & $1 / 000$ & 0.787 \\
\hline Angiogenesis & 0.998 & 0.998 & 0.998 & $1 / 000$ & $1 / 000$ & 0.556 \\
\hline
\end{tabular}

*Statistically significant

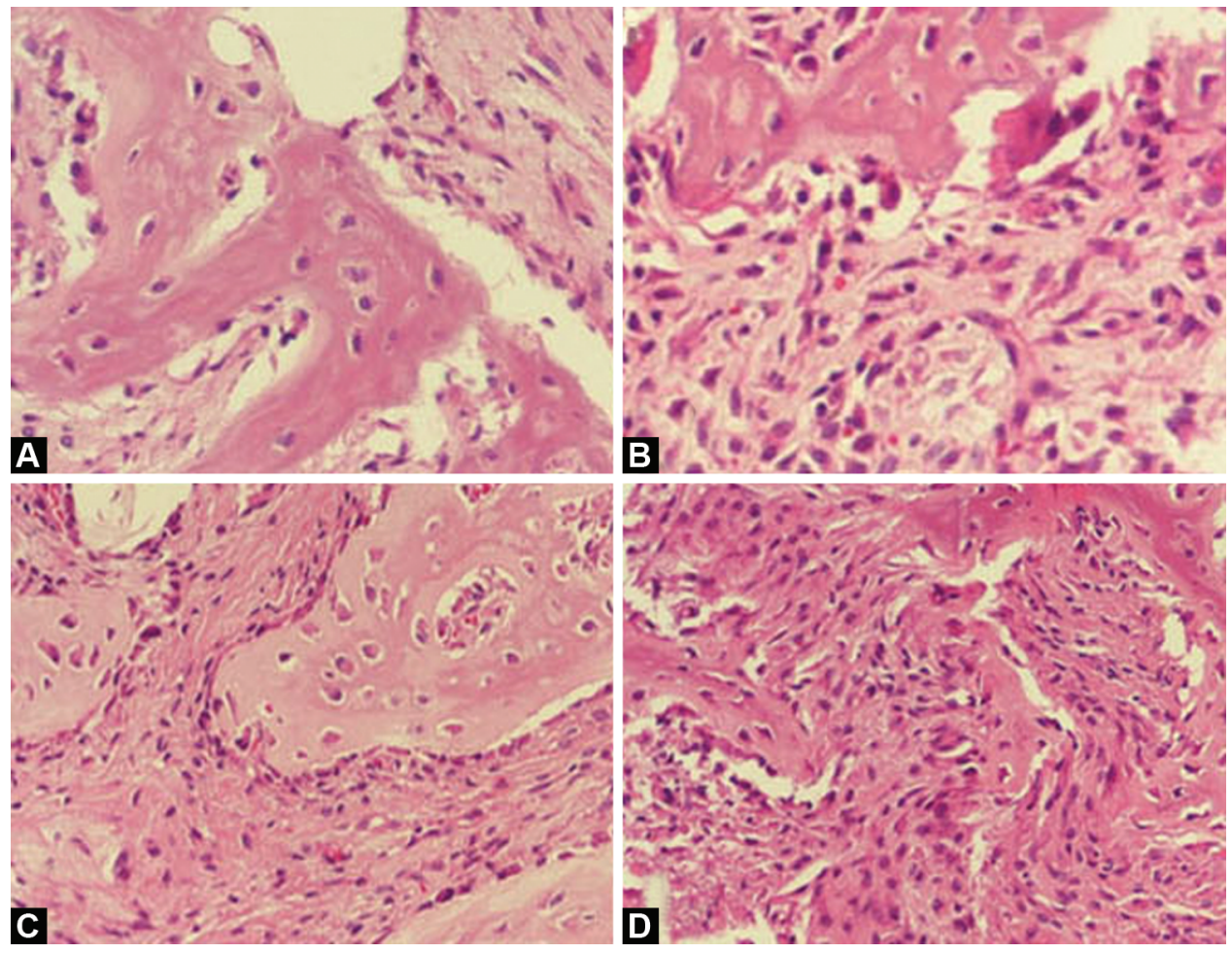

Figs 3A to D: Histopathology of the case (A) and control (B) groups on the 3rd day and the case (C) and control (D) groups on the 7th day. On the 3rd day, the control group (B) showed the inflammation with osteoclasts and also connective tissue. The laser group (A) images showed trabecular thin bone with osteoblasts and connective tissue. On the 7th day, the control and laser groups (C) and (D) showed the regular bone formation with lots of osteoclasts 
0.05). In addition, the number of fibroblasts increased and the number of neutrophils decreased in the 5 minute radiation group in comparison with the control group, but the differences were not statistically significant ( $p$ value $>0.05$ ) (Fig. 3).

\section{Discussion}

Tooth extraction can be accompanied by complications such as swelling, trismus, and pain. ${ }^{14}$ The best treatment is prevention. Among those that can accelerate wound healing are low-level lasers. The effect of the low-level laser depends on several factors like the time of radiation. ${ }^{5}$

Based on our findings, there was no significant difference in the increase in laser radiation time in angiogenesis on the 3rd and 7th days in comparison with the control group. Contrary to the results of the study, Maiya et al., ${ }^{15}$ who performed a low-level laser treatment for diabetic wound healing, concluded that low-level laser was effective in increasing angiogenesis, and the rate of angiogenesis in the case group was started earlier than the control group. Ozcelik et al. ${ }^{16}$ also showed that use of a low-level laser could increase the formation of blood vessels and wound healing. This difference can be due to differences in the type of laser, its parameters, the type of tissue examined in the study, or the method of implementation.

In this study, the percentage of macrophages in the 3rd day increased in all groups compared the control group, which was significant in the 5 minute radiation group. These macrophages increase cellular migration and subsequently accelerate wound healing by secreting cytokines. In addition, use of a low-level laser reduced the number of neutrophils on the 3rd day and lymphocytes and macrophages on the seventh day in the studied groups compared to the control group, but the difference was not statistically significant. Similar to the current study, Pereira et al. ${ }^{17}$ concluded that low-level laser radiation reduced inflammation, stimulated the cell division of inflammatory cells, and improved bone healing.

Contrary to the current study, Lopez-Ramirez et al..$^{10}$ explored the effect of a low-level laser on pain control, facial swelling, and trismus following mandibular third molar surgery. The results showed that laser application with the parameters used in this study had no effect on pain reduction, swelling, and trismus after removing the impacted third molars. That may be due to the difference in the results generated by laser parameters used and its human application.

Based on our findings, low-level laser radiation increased the number of fibroblasts on the 3 rd day compared to the control group (except for the 10 minute radiation group), but these differences were not statistically significant.

Silveira et al. ${ }^{18}$ assessed the efficacy of low-level laser radiation with different wavelengths in wound healing in animals and demonstrated that local application of low-level laser accelerated the wound healing process probably due to increased collagen synthesis, confirming the results of the present study.

Our results showed that osteogenicity increased on day 3 with an increase in laser radiation time (except for the 10 minute radiation group), which was significant only in the 5 minute laser radiation group. In addition, osteogenesis increased on the 7th day in all laser radiation groups compared to the control group, but the difference was not statistically significant. Similar to the results of the present study, Stein et al. ${ }^{19}$ reported that the use of the helium-neon laser increased the number of osteoblasts via an increase in the osteogenic markers.
Park et al. ${ }^{8}$ examined the effect of increasing the low-level diode laser radiation time on the expression of the bone healing genes. The highest expression of these genes was observed in the treatment group with a 5 minute laser radiation for 7 days. These results showed that laser therapy had a time-dependent effect.

In a study on 20 rats, Pretel et al. ${ }^{9}$ examined the effect of a low-level laser on the bone loss healing. The results showed that laser irradiation groups had a lower rate of inflammation and bone formation on days 15 and 45 in comparison with the control group. There was also no significant difference between the two groups in day 60, which was similar to the present study.

Diodes lasers are small, high-performance, and low-cost, making them an ideal device for dentists and maxillofacial surgeons. $^{20}$

The limitation of this study was the absence of different types of lasers to compare their effects with each other.

\section{Conclusion}

Low-level laser radiation could accelerate the tooth socket healing process, which was particularly noticeable in the 5 minute radiation over 3 days.

Using a low-level laser can be helpful in accelerating the healing of the tooth socket and reduce the complications after tooth extraction.

\section{References}

1. Mergoni G, Vescovi P, Passerini P, et al. Effects of zoledronic acid and dexamethasone on early phases of socket healing after tooth extraction in rats: a preliminary macroscopic and microscopic quantitative study. Med Oral Patol Oral Cir Bucal 2019;24(3):339-345. DOI: $10.4317 /$ medoral.22883.

2. Mahattanadul S, Mustafa MW, Kuadkaew S, et al. Oral ulcer healing and anti-candida efficacy of an alcohol-free chitosan-curcumin mouthwash. Eur Rev Med Pharmacol Sci 2018;22(20):7020-7023.

3. Beayens TA. Wound care guidelines and formulary for community nurses. J Wound Care 2000;9(3):106-108. DOI: 10.12968/ jowc.2000.9.3.25963.

4. Klein M, de Quadros De Bortolli J, Guimaraes FS, et al. Effects of cannabidiol, a cannabis sativa constituent, on oral wound healing process in rats: clinical and histological evaluation. Phytother Res 2018;32(11):2275-2281. DOI: 10.1002/ptr.6165.

5. Aras $M H$, Gungormus $M$. The effect of low-level laser therapy on trismus and facial swelling following surgical extraction of a lower third molar. Photomed Laser Surg 2009;27(1):21-24. DOI: 10.1089/ pho.2008.2258.

6. Cruz DR, Kohara EK, Ribeiro MS, et al. Effects of low-intensity of laser therapy on the orthodontic movement velocity of human teeth: a preliminary study. Lasers Surg Med 2004;35(2):117-120.DOI: 10.1002/ Ism.20076.

7. Tunc SK, Yayli NZ, Talmac AC, et al. Clinical comparison of the use of ER,CR:YSGG and diode lasers in second stage implants surgery. Saudi Med J 2019;40(5):490-498. DOI: 10.15537/smj.2019.5.24105.

8. Park JB, Ahn S-J, Kang Y-G, et al. Effects of increased low-level diode laser irradiation time on extraction socket healing in rats. Lasers Med Sci 2013;30(2):719-726. DOI: 10.1007/s10103-013-1402-6.

9. Pretel H, Lizarelli RF, Ramalho LT. Effect of low-level laser therapy on bone repair: histological study in rats. Lasers Surg Med 2007;39(10):788-796. DOI: 10.1002/Ism.20585.

10. Lopez-Ramirez M, Vilchez-Perez MA, Gargallo-Albiol J, et al. Efficacy of low-level laser therapy in the management of pain, facial swelling, and postoperative trismus after a lower third molar extraction. A preliminary study. Lasers Med Sci 2012;27(3):559-566. DOI: 10.1007/ s10103-011-0936-8. 
11. Goymen $M$, Isman E, Taner L, et al. Histomorphometric evaluation of the effects of various diode lasers and force levels on orthodontic mini screw stability. Photomed Laser Surg 2015;33(1):29-34. DOI: 10.1089/pho.2014.3833.

12. Guzzardella GA, Torricelli P, Roberto Giardino N. Osseointegration of endosseous ceramic implants after postoperative lowpower laser stimulation: an in vivo comparative study. Clin Oral Impl Res 2003;14(2):226-232. DOI: 10.1034/j.1600-0501.2003. 00872.x.

13. Peimani A, Sardary F. Effect of low-level laser on healing of temporomandibular joint osteoarthritis in rats. J Dent (Tehran) 2014;11(3):319-327.

14. da Silva JP, da Silva MA, Almeida AP, et al. Laser therapy in the tissue repair process: a literature review. Photomed Laser Surg 2010;28(1):1721. DOI: $10.1089 /$ pho.2008.2372.

15. Maiya GA, Kumar P, Rao L. Effect of low intensity helium-neon(He-Ne) laser irradiation on diabetic wound healing dynamics. Photomed Laser Surg 2005;23(2):187-190. DOI: 10.1089/pho.2005.23.187.
16. Ozcelik O, Cenk Haytac M, Kunin A, et al. Improved wound healing by low-level laser irradiation after gingivectomy operations: a controlled clinical pilot study. Med Sci 2009;44(2):123-126.

17. Pereira CL, Sallum EA, Nociti Jr. FH, et al. The effect of low-intensity laser therapy on bone healing around titanium implants: a histometric study in rabbits. Int J oral Maxillofac Imp 2009;24(1):47-51.

18. Silveira PC, Silva LA, Fereitas TP, et al. Effect of low-power laser irradiation (LPLI) at different wavelengths and doses on oxidative stress and fibrogenesis parameters in an animal model of wound healing. Laser Med Sci 2011;26(7):125-131. DOI: 10.1007/s10103-0100839-0.

19. Stein A, Benayahu D, Maltz $L$, et al. Low-level laser irradiation promotes proliferation and differentiation of human osteoblasts in vitro. Photomed Laser Surg 2005;23(2):161-166. DOI: 10.1089/ pho.2005.23.161.

20. Deppe $\mathrm{H}$, Horch HH. Laser applications in oral surgery and implant dentistry. Lasers Med Sci 2007;22(4):217-221. DOI: 10.1007/s10103007-0440-3. 